EPJ Web of Conferences 59, 08007 (2013)

DOI: $10.1051 /$ epjconf/20135908007

(C) Owned by the authors, published by EDP Sciences, 2013

\title{
Wavefront performance analysis of Laser MégaJoule optical components
}

\author{
S. Mainguy ${ }^{\mathrm{a}}$ and L. Le Déroff \\ CEA, DAM, CESTA, BP. 2, 33114 Le Barp, France
}

\begin{abstract}
We present a review of recent analysis of optical specifications for the Laser Mégajoule (LMJ) plain size $(40 \mathrm{~cm} \times 40 \mathrm{~cm})$ components. Our studies are mainly achieved using the Miró laser propagation code. The purpose of this paper is first to describe the different methods used to model the wavefronts transmitted by the components. Then, we give examples of evaluation results for the following issues: surface imperfection specification on the $1 \omega$ phase plate, surface conditioning defects tolerance on $1 \omega$ transport mirrors, Power Spectral Distribution (PSD) specifications of the transmitted wavefront and of the roughness on the components of the amplifying section.
\end{abstract}

\section{CONTEXT AND OBJECTIVES}

The Laser Mégajoule (LMJ) [1] has about 40 large optics per beam and, for 22 bundles, about 7.000 optical components. Therefore a set of acceptance criteria is needed when the optical components are exceeding the specifications (Figure 1). This set of rules is critical even for a small nonconformance ratio.

The goals of the corresponding impact analyses (Figure 2) are to check, for all delivered components, the following laser performance criteria:

- in the amplifying section: beam output energy, peak fluences $\mathrm{F}_{\text {peak }}$ on all the components (especially L3 spatial filter lens), near-field amplitude and phase structures (M1 and M2 mirrors);

- in the transport-frequency conversion-focusing sections: beam modulation depth $\left(\mathrm{F}_{\text {peak }} /<\mathrm{F}>\right.$ ), peak fluences and intensities, breakup integral, near-field amplitude and phase structures (transport mirrors, KDP crystal converters), $3 \omega$ focal spot dimension.

The laser performance analysis is mainly conducted through numerical simulations with the Miró laser propagation code $[2,3]$. Current LMJ components of interest are: laser amplification slabs, spatial filter lens, transport mirrors, $1 \omega$ and $3 \omega$ gratings, KDP (Pockel cells and frequency conversion).

\section{WAVEFRONT MODELING METHODS}

In the numerical simulations with the Miró code, component wavefront and roughness are obtained from measurements or models. Three methods are currently used to achieve this modeling:

- analytical expressions allow to study the propagation downstream local defects and defect densities; defects nature can be either amplitude or phase and their geometries are various like Gaussian, disk, etc. Also the propagation of residual 1D or 2D periodic aberrations along the chain can be analytically treated: for example, a sinusoidal residual phase is used to model inter-actuator defects on an adaptative mirror [4].

\footnotetext{
${ }^{a}$ e-mail: stephane.mainguy@cea.fr
}

This is an Open Access article distributed under the terms of the Creative Commons Attribution License 2.0, which permits unrestricted use, distribution, and reproduction in any medium, provided the original work is properly cited. 


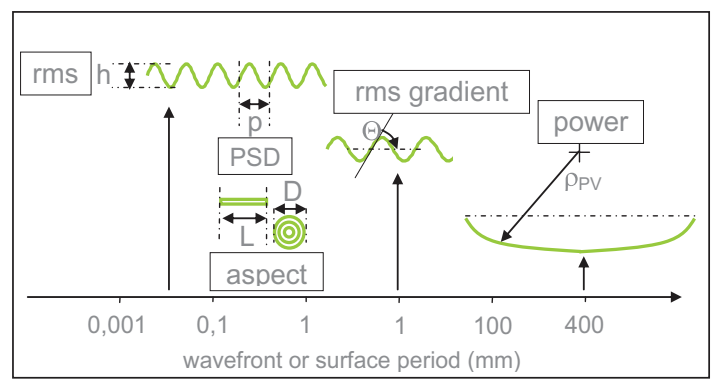

Figure 1. Optical specifications of LMJ components, as a function of wavefront/surface period.

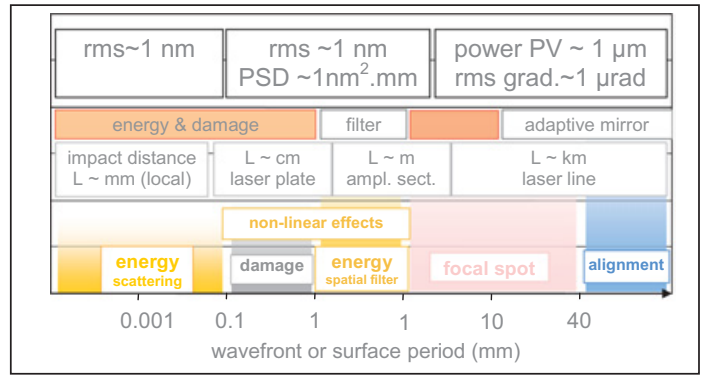

Figure 2. Optical specifications values and their impact on the laser performance, as a function of wavefront/surface period.

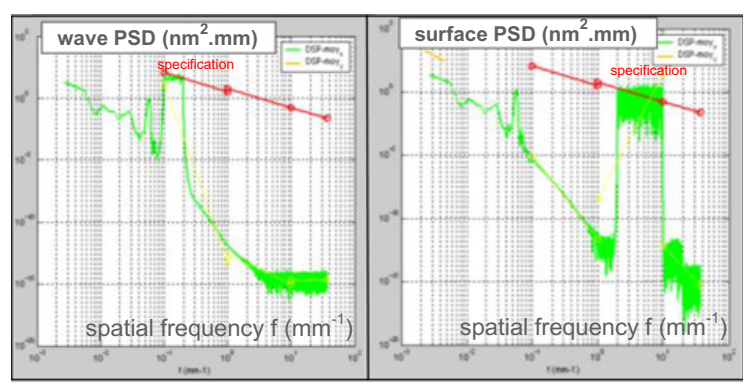

Figure 3. Left: the wave PSD of a $1 \omega$ wavefront generated with ALEA for parameters : $A=(2 \pi / \lambda) \times$ (wave rms $2 \mathrm{~nm})=12 \mathrm{mrad}, \mathrm{p}_{\min }=(\sqrt{2} / 2 \pi) \times($ band min. period $5 \mathrm{~mm}), \mathrm{p}_{\max }=(\sqrt{2} / 2 \pi) \times($ band max. period $10 \mathrm{~mm})$. Right: the roughness PSD obtained from a roughness generated with ALEA.

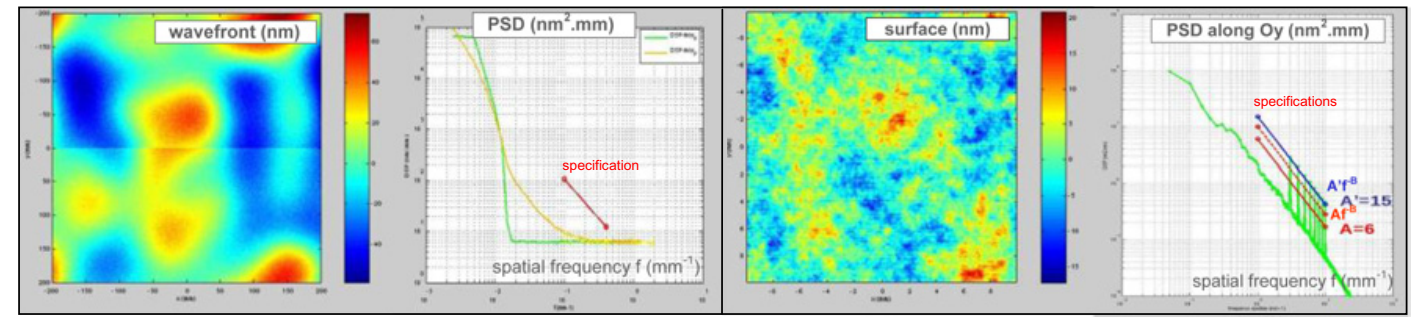

Figure 4. Left: a $40 \mathrm{~cm} \times 40 \mathrm{~cm}$ wavefront with a systematically centered phase defect (due to material), as observed on a few amplification plates. Right: a $20 \mathrm{~mm} \times 20 \mathrm{~mm}$ surface with PSD peaks on band $0.1-1 \mathrm{~mm}$, as observed on some polished KDP crystals.

- the random phase function $\operatorname{ALEA}\left(\mathrm{A}, \mathrm{p}_{\min }, \mathrm{p}_{\max }\right)$ of the Miró code is very useful to simulate the propagation in presence of component wavefront or roughness errors, defined by a uniform Power Spectral Distribution (PSD) on critical spatial period bands [ $\mathrm{p}_{\min }, \mathrm{p}_{\max }$ ] (examples on Figure 3 ).

- random phase maps can be numerically generated using a new function to be integrated into the Miró code, based on a Fourier method. This method allows to study the propagation with a component specific wavefront or roughness (examples on Figure 4). 


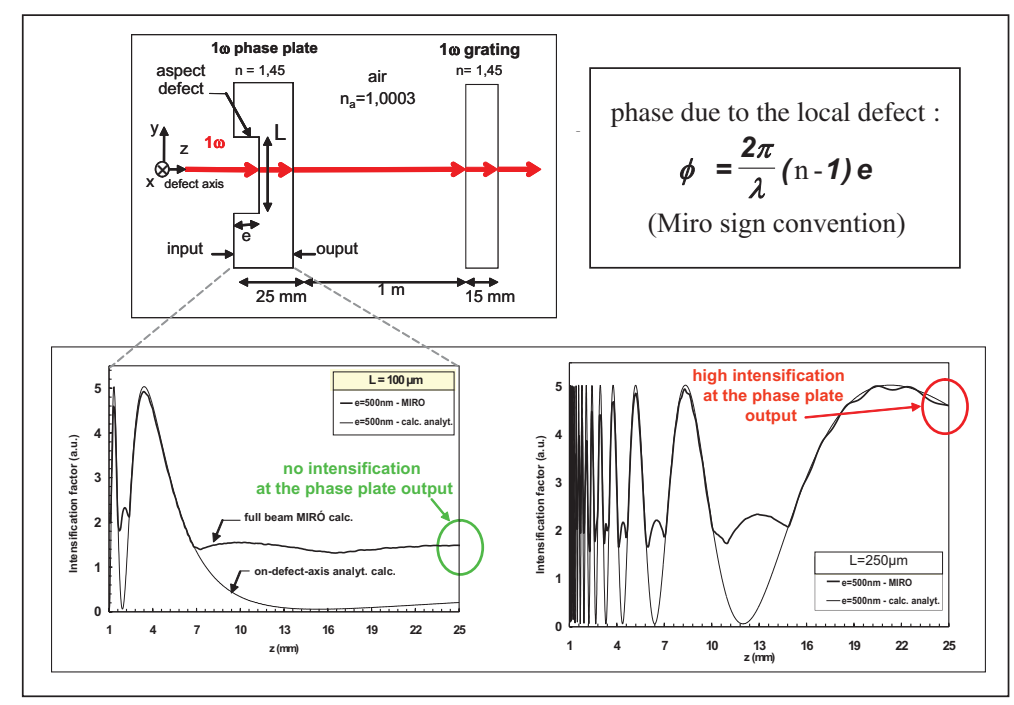

Figure 5. Modulation calculations downstream a model surface imperfection located at the input of the LMJ $1 \omega$ phase plate: comparison of ISO10110-7 specifications $\mathrm{L}=100 \mu \mathrm{m}$ and $\mathrm{L}=250 \mu \mathrm{m}$, for a maximum defect depth $\mathrm{e}=500 \mathrm{~nm}$. On-axis analytical expression is obtained from [5].

\section{EVALUATION OF SURFACE IMPERFECTION AND TECHNOLOGICAL DEFECTS}

\subsection{Evaluation of a surface imperfection on the LMJ $1 \omega$ phase plate}

Downstream amplitude modulations are modeled with a defect (disk, width $\mathrm{L}$, depth e) located at the input of the LMJ $1 \omega$ phase plate. ISO10110-7 specifications $\mathrm{L}=100 \mu \mathrm{m}$ and $\mathrm{L}=250 \mu \mathrm{m}$ are compared using propagation calculations on the inside of the phase plate for a maximum defect depth $\mathrm{e}=500 \mathrm{~nm}$ (Figure 5): the $\mathrm{L}=250 \mu \mathrm{m}$ specification is not acceptable.

The 1D downstream propagation is then simulated for one LMJ beam at nominal energy and for specification $\mathrm{L}=100 \mu \mathrm{m}$ : the beam modulations increase strongly with the defect depth, due to non-linear effects in the $3 \omega$ components. For specification $\mathrm{L}=100 \mu \mathrm{m}$ and an intermediate depth $\mathrm{e}=100 \mathrm{~nm}$, the increase of the modulations on $3 \omega$ components is acceptable $(+4 \%$ to $+15 \%)$.

\subsection{Tolerance on density of Gaussian conditioning scalds on $1 \omega$ transport mirrors}

Full-beam propagation is simulated downstream Gaussian conditioning scalds located on $1 \omega$ transport mirrors. Scald densities of $0.1 / \mathrm{cm}^{2}$ and $3 / \mathrm{cm}^{2}$ with maximum size (width $\mathrm{D}_{1 / \mathrm{e}^{2}}=500 \mu \mathrm{m}$, depth $\mathrm{h}=200 \mathrm{~nm}$ ) are studied. For a scald density of $3 / \mathrm{cm}^{2}$, the beam modulation depth increases up to $+18-33 \%$ and the peak fluences are close to damage threshold: this density is not acceptable. A scald density of $0.1 / \mathrm{cm}^{2}$ is acceptable.

\section{EVALUATION OF WAVEFRONT/ROUGHNESS PSD AND RMS SPECIFICATIONS IN THE AMPLIFYING SECTION}

The propagation of $1 \omega$ wavefront PSD periods $\mathrm{p}=1-10 \mathrm{~mm}$, located on each component in the LMJ amplifying section, are analyzed. The criteria mentioned in paragraph 1 are considered (Figure 6).

Considering the $\mathrm{F}_{\text {peak }}$ criteria on lens L3, PSD values up to 0.8 time the specification on each component of the amplifying section are valid, in the most critical spatial band (1-5 mm). 


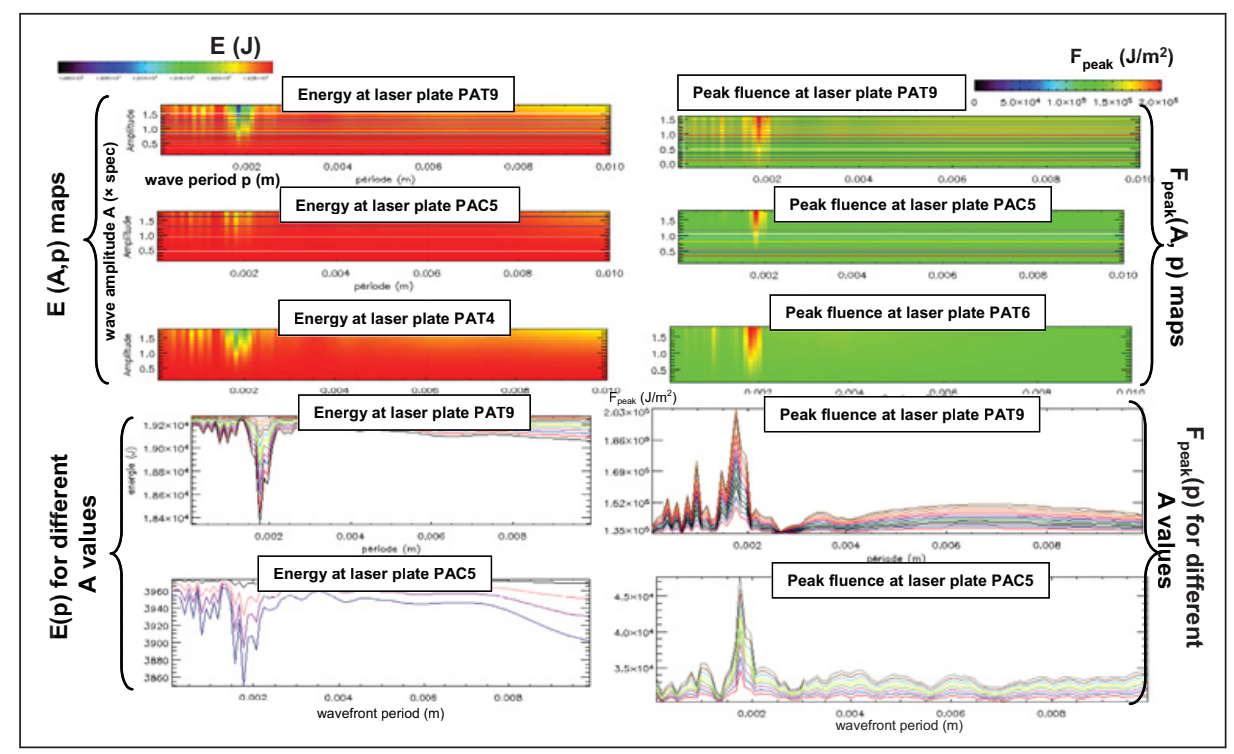

Figure 6. Analysis of $1 \omega$ energy $E$ and peak fluences $F_{\text {peak }}$ at laser plates in the LMJ amplifying section as a function of $1 \omega$ wavefront PSD periods $\mathrm{p}=1-10 \mathrm{~mm}$ located on each plate. Amplitude $\mathrm{A}=1$ corresponds to the laser plate PSD specification.

Also, roughness PSD on band 0.1-1 mm on the KDP crystals of the $1 \omega$ Pockels cells (PEPC) is studied. No critical impact is obtained on the PEPC window: the modulation rate at window input is $+6 \%$ and fluences at the 4 th pass $\left(\sim 4 \mathrm{~J} / \mathrm{cm}^{2}\right)$ are far below the damage threshold. Moreover, the $0.1-$ $1 \mathrm{~mm}$ band is not critical in the amplifying section thanks to spatial filtering and because there is no non-linear perturbation of the gain during laser amplification for such band.

\section{CONCLUSION}

The wavefront performance analysis of the LMJ components requires an intensive use of numerical simulations with the Miró laser propagation code. Future works concern the evaluation of $3 \omega$ end-ofline component specifications: roughness imperfections, PSD and rms. We intend to evaluate the reallocation of wavefont rms gradient in the transport due to possible compensations between mechanical and static aberrations.

The authors thank all collaborators at CEA-CESTA: V. Beau, E. Bordenave, C. Chappuis, S. Vermersch, J.-P. Airiau, P. Cormont, N. Darbois, T. Lanternier, E. Lavastre, M. Mangeant, C. Maunier, D. Taroux, J. Néauport, S. Bouillet, G. Gaborit, J. Daurios, G. Razé, T. Bart, V. Denis, C. Grosset-Grange, E. Journot, C. Rouyer, N. Ferriou-Daurios, S. Seznec.

\section{References}

[1] J. Ebrardt, J.-M. Chaput, 6th IFSA conf., J. of Physics: Conference Series, 244, 032017 (2010)

[2] O. Morice, Optical Engineering, Vol. 42, No. 6, 1530-1541 (June 2003)

[3] contacts: codemiro@cea.fr

[4] S. Mainguy, C. Grosset-Grange, E. Bordenave, 6th IFSA conf., J. of Physics: Conference Series, 244, 032021 (2010)

[5] S. Mainguy, I. Tovena-Pecault, B. Le Garrec, Proc. of SPIE, Vol. 5991, 59910G (2005) 